\title{
SOME REMARKS ON THE CRITICAL POINT THEORY
}

\author{
Chong Li
}

Dedicated to Professor Louis Nirenberg on the occasion of his 80th birthday

ABstract. In this paper we discuss some problems about critical point theory. In the first part of the paper we study existence and multiplicity results of semilinear second order elliptic equation:

$$
\begin{cases}-\Delta u=f(x, u) & \text { for } x \in \Omega, \\ u=0 & \text { for } x \in \partial \Omega\end{cases}
$$

In [4], the authors study the contractibility of level sets of functionals associated with some elliptic boundary value problems. In this paper by using Morse theory and minimax method we give a more precise description of topological construction of level set of critical value of energy functional for mountain pass type critical point. It is well known that nondegenerate critical point is isolated, so if a critical point is not isolated, it must be a degenerate critical point. In the second part we will give an example that all the critical points of functional of a class of oscillating equation with Neumann boundary condition are isolated and the equation has only constant solutions. Moreover, critical groups of each critical point of the functional are trivial. The elliptic sine-Gordon equation originates from the static case of the hyperbolic sine-Gordon equation modelling the Josephson junction in superconductivity, which is of contemporary interest to physicists. The problem is similar to the elliptic sine-Gordon equation so we believe that it derives from profound physical backdrop.

2000 Mathematics Subject Classification. 35J65, 58E05.

Key words and phrases. Mountain pass theorem, order intervals, Morse theory, minimax method.

Supported by NSFC(10601058), NSFC(10471098), NSFC(10571096) and TYF(10526027). 


\section{Introduction}

We consider elliptic equation with Dirichlet boundary condition:

$$
\begin{cases}-\Delta u=f(x, u) & \text { for } x \in \Omega, \\ u=0 & \text { for } x \in \partial \Omega,\end{cases}
$$

Let $\Omega \subset \mathbb{R}^{\mathbb{N}}$ be a bounded domain with smooth boundary $\partial \Omega$.

Denote by $\sigma(-\Delta)=\left\{\lambda_{i} \mid 0<\lambda_{1}<\lambda_{2} \leq \ldots \leq \lambda_{k} \leq \ldots\right\}$ the eigenvalues of the following linear problem:

$$
\begin{cases}-\Delta u=\lambda u & \text { for } x \in \Omega, \\ u=0 & \text { for } x \in \partial \Omega .\end{cases}
$$

We assume that $f \in C^{1}(\mathbb{R}, \mathbb{R})$ and $f(0)=0$. Let

$$
b=\lim _{t \rightarrow 0_{+}} \frac{f(x, t)}{t}, \quad a=\lim _{t \rightarrow 0_{-}} \frac{f(x, t)}{t}, \quad b \neq a, \quad F(x, u)=\int_{0}^{u} f(x, s) d s .
$$

In view of the variational point, solutions of (1.1) are critical points of corresponding functional defined on the Hilbert space $\mathrm{E}=H_{0}^{1}(\Omega)$.

Let $\mathrm{X}=C_{0}^{1}(\Omega) \triangleq\left\{u \in C^{1}(\Omega) \mid u(x)=0, x \in \partial \Omega\right\}$. It is a Banach space, and define $\left[u_{1}, u_{2}\right] \triangleq\left\{u \in \mathrm{X} \mid u_{1} \leq u \leq u_{2}, x \in \Omega\right\}$ be the order interval in X.

Consider the functional

$$
J(u)=\frac{1}{2} \int_{\Omega}|\nabla u|^{2} d x-\int_{\Omega} F(x, u) d x .
$$

Assume the following conditions:

(i) There are constant $C>0$ such that

$$
\left|f_{t}^{\prime}(x, t)\right| \leq C\left(1+|t|^{p-1}\right), \quad t \in \mathbb{R} \backslash\{0\},
$$

where $1<p<2^{*}-1,2^{*}=2 N /(N-2)$, if $N \geq 3$, and $2^{*}=\infty$, if $N=1,2$.

(ii) There exists $M_{1}>0, M_{2}<0$ such that $f\left(x, M_{1}\right)<0, f\left(x, M_{2}\right)>0$ for $x \in \Omega$.

(iii) $\lambda_{l-1} t^{2} \leq 2 F(x, t) \leq \underline{a}\left(t^{-}\right)^{2}+\underline{b}\left(t^{+}\right)^{2},|t| \leq \delta,(\underline{a}, \underline{b}) \in C_{l 1} \cap Q_{l}, l \geq 3$, and

$$
\begin{aligned}
& \lambda_{l-1} \leq \frac{f(x, t)}{t} \leq \underline{b}, \quad 0 \leq t \leq \delta, \\
& \lambda_{l-1} \leq \frac{f(x, t)}{t} \leq \underline{a}, \quad-\delta \leq t \leq 0,
\end{aligned}
$$

and 0 is a isolated critical point of $J$. 
(iv) $\bar{a}\left(t^{-}\right)^{2}+\bar{b}\left(t^{+}\right)^{2} \leq 2 F(x, t) \leq \lambda_{l+1} t^{2},|t| \leq \delta,(\bar{a}, \bar{b}) \in C_{l 2} \cap Q_{l}$, and

$$
\begin{aligned}
& \bar{b} \leq \frac{f(x, t)}{t} \leq \lambda_{l+1}, \quad 0 \leq t \leq \delta, \\
& \bar{a} \leq \frac{f(x, t)}{t} \leq \lambda_{l+1}, \quad-\delta \leq t \leq 0,
\end{aligned}
$$

$\min \{\bar{a}, \bar{b}\} \geq \lambda_{2}$ and 0 is a isolated critical point of $J$.

Here $C_{l 1}, C_{l 2}$ are two strictly decreasing continuous curves defined in [5], $Q_{l}=\left(\lambda_{l-1}, \lambda_{l+1}\right)^{2}$.

Now we state our main abstract theorem:

TheOrem 1.1. Let $\mathrm{E}$ be a Banach space. Functional $J$ is defined on $\mathrm{E}$. $J \in C^{1}(\mathrm{E}, \mathbb{R})$ satisfies (PS) condition. $J$ is bounded from below on $\mathrm{E}$. And for the mountain pass type critical points $u$ of $J$, its critical group $C_{q}(J, u)$ is of the form $\delta_{q 1} G$. Assume $u_{1}, u_{2}$ and $u_{3}$ are three critical points of $J$, where $u_{1}, u_{2}$ are local minimizers and $u_{3}$ is a mountain pass point, and $J\left(u_{3}\right)>\max \left\{J\left(u_{1}\right), J\left(u_{2}\right)\right\}$, $J\left(u_{1}\right)=\inf _{u \in \mathrm{E}} J(u)<\infty$. Define $c_{i}=J\left(u_{i}\right), i=1,2,3$. If $J$ has only three critical points in $J^{c_{3}}$, then either

(a) $H_{q}\left(J^{c_{3}}\right) \cong \delta_{q 0} G$;

or

(b) $H_{q}\left(J^{c_{3}}\right)= \begin{cases}G \oplus G & \text { if } q=0, \\ G & \text { if } q=1, \\ 0 & \text { if } q \geq 2 .\end{cases}$

The following proposition makes an application of Theorem 1.1:

Proposition 1.2.

(a) Under the hypothesis (i)-(iii), (1.1) admits four nontrivial solutions.

(b) Under the hypothesis (i), (ii), (iv), there exists at least four nontrivial solutions of (1.1).

As a matter of fact, we are inclined to generalizing Proposition 1.2 to general case. By giving the additional condition:

(v) there exists $q_{0} \geq 2$ such that $C_{q_{0}}(J, 0) \neq 0$,

we have:

Proposition 1.3. If there exist two pairs sub-sup solution of (1.1) nearby 0 and with the assumption (v) we could obtain at least four nontrivial solutions.

In many cases if we assume that 0 is a nondegenerate critical point of $J$ or $J$ has a local linking at 0 and critical groups $C_{q}(J, 0)$ is clear by some technical methods, and also assume that there exist two pairs of sub-sup solution nearby 0 , then by mountain pass theorem in order intervals [3], we can get a sign-changing 
mountain pass point, and by degree theory we obtain the second sign-changing solutions. In this paper we will show that if critical group $C_{q}(J, 0)$ is nontrivial for some $q$, then by comparing the difference of topological construction of different level sets of energy functional $J$, we still verify the existence of four nontrivial solutions even local degree of 0 is zero. However, we could not describe the property of the fourth solution we get.

In Section 3 we give the proof of Theorem 1.1. It is well known that nondegenerate critical point is isolated, so if a critical point is not isolated so it must be a degenerate critical point. In Section 4 we will give an example that all the critical points of functional of a class of oscillating equation with Neumann boundary condition are degenerate and also are isolated. Moreover, critical groups of each critical point of the functional are trivial. First, we recall some notions and known results on critical point theory in Section 2.

\section{Preliminaries}

Let $E$ be a Banach space, $J \in C^{1}(\mathrm{E}, \mathbb{R}), K=\left\{u \in \mathrm{E} \mid J^{\prime}(u)=0\right\}$, $J^{c}=\{u \in \mathrm{E} \mid J(u) \leq c\}, K_{c}=\{u \in K \mid J(u)=c\}, c \in \mathbb{R}$.

Definition 2.1. We say that $J$ satisfies the $(\mathrm{PS})_{c}$ condition if any sequence $\left\{u_{k}\right\} \subset$ E along with $J\left(u_{k}\right) \rightarrow c$ and $J^{\prime}\left(u_{k}\right) \rightarrow 0(k \rightarrow$ infty $)$ possesses a convergent subsequence. We say that $J$ satisfies the (PS) condition if it satisfies $(\mathrm{PS})_{c}$ for all $c \in \mathbb{R}$.

Definition 2.2. Assume that $J \in C^{1}(\mathrm{E}, \mathbb{R}), c \in \mathbb{R} . N$ is a closed neighbourhood of $K_{c}$. If for all $\varepsilon^{*}>0$ and all $N$, there exists $\varepsilon \in\left(0, \varepsilon^{*}\right)$ and a continuous map $\eta:[0,1] \times \mathrm{E} \rightarrow \mathrm{E}$, such that:

(a) $\eta(0, \cdot)=\mathrm{id}$,

(b) $\eta(t, u)=u$ for all $u \notin J^{-1}\left[c-\varepsilon^{*}, c+\varepsilon^{*}\right]=\left\{u \in \mathrm{E} \mid c-\varepsilon^{*} \leq J(u) \leq\right.$ $\left.c+\varepsilon^{*}\right\}$

(c) $J(\eta(\cdot, u))$ is nonincreasing, for all $u \in \mathrm{E}$,

(d) $\eta\left(1, J^{c+\varepsilon} \backslash N\right) \subset J^{c-\varepsilon}$.

Then we say that $J$ satisfies deformation property.

By using the famous deformation theorem, we know that $J$ satisfies deformation property when $J$ satisfies (PS) condition.

Let $\mathrm{E}$ be a Hilbert space, $\mathrm{P}_{\mathrm{E}}$ is a closed convex cone of $\mathrm{E}$. $\mathrm{X}$ is densely imbedded to E. Assume that $\mathrm{P}=\mathrm{X} \cap \mathrm{P}_{\mathrm{E}}$ and $\stackrel{\circ}{\mathrm{P}}$, the interior of $\mathrm{P}$ is nonempty. We assume that any order interval is bounded in any finite dimensional subspace of X.

The functional $J: \mathrm{E} \rightarrow \mathbb{R}$ satisfies the following conditions:

(A) $J \in C^{2}(\mathrm{E}, \mathbb{R})$ and satisfies (PS) condition in $\mathrm{E}$ and deformation property in X. $J$ only has finitely many isolated critical points. 
(B) The gradient of $J$ is of the form $\nabla J=\mathrm{id}-\mathrm{K}_{\mathrm{E}}$, where $\mathrm{K}_{\mathrm{E}}$ : $\mathrm{E} \rightarrow \mathrm{E}$ is compact. $\mathrm{K}_{\mathrm{E}}(\mathrm{X}) \subset \mathrm{X}$ and the restriction $\mathrm{K}=\left.\mathrm{K}_{\mathrm{E}}\right|_{\mathrm{X}}: \mathrm{X} \rightarrow \mathrm{X}$ is of class $C^{1}$ and strongly preserving, i.e. $\mathrm{K}(u) \gg \mathrm{K}(v)$ if $u \gg v, u \gg v$ if and only if $u-v \in \stackrel{\circ}{\mathrm{P}}$.

(C) $J$ is bounded from below on any order interval in X.

Now, we state the mountain pass theorem in order intervals (see [3]) as follows:

Theorem 2.3 (Mountain pass theorem in order intervals). Suppose $J$ satisfies (A)-(C) and $\left\{v_{1}, v_{2}\right\},\left\{w_{1}, w_{2}\right\}$ are two pairs of strict sub-supsolution of $\nabla J=0$ in $\mathrm{X}$ with $v_{1}<w_{2},\left[v_{1}, v_{2}\right] \cap\left[w_{1}, w_{2}\right]=\emptyset$. Then $J$ has a mountain pass point $u_{0}, u_{0} \in\left[v_{1}, w_{2}\right] \backslash\left(\left[v_{1}, v_{2}\right] \cup\left[w_{1}, w_{2}\right]\right)$. More precisely, let $v_{0}$ be the maximal minimizer of $J$ in $\left[v_{1}, v_{2}\right]$ and $w_{0}$ be the minimal minimizer of $J$ in $\left[w_{1}, w_{2}\right]$. Then $v_{0}<u_{0}<w_{0}$. Moreover, $C_{1}\left(J, u_{0}\right)$, the 1 -th critical group of $J$ at $u_{0}$, is nontrivial.

REMARK 2.4. Theorem 2.3 still holds if $J \in C^{1}(\mathrm{E}, \mathbb{R})$ and $\mathrm{K}$ is of class $C^{0}$.

REMARK 2.5. Theorem 2.3 still holds if $J$ has infinitely many isolated critical points.

Now, we recall some notions and known results on Morse theory. Assume that $\mathrm{E}$ is a Hilbert space with inner product $\langle\cdot, \cdot\rangle$ and corresponding norm $\|\cdot\|$, functional $J \in C^{1}(\mathrm{E}, \mathbb{R})$. Let $u_{0}$ be an isolated critical point of $J$ with $J\left(u_{0}\right)=c, c \in \mathbb{R}$, and $U$ be a neighbourhood of $u_{0}$ such that $K \cap U=\left\{u_{0}\right\}$ being $K=\left\{u \in E \mid J^{\prime}(u)=0\right\}$ the critical set of $J$. Then we call

$$
C_{q}(J, 0)=H_{q}\left(J^{c} \cap U, J^{c} \cap U \backslash\left\{u_{0}\right\}, G\right), \quad q=0,1, \ldots
$$

the $q$-th critical group, with coefficient group $G$ of $J$ at $u_{0}$, where $J^{c}=\{u \in \mathrm{E} \mid$ $J(u) \leq c\} . H_{q}(A, B ; G)$ stands for the $q$-th singular relative homology group of the topogical pair $(A, B)$ with coefficients in an Abelian group $G$, from now on we denote it by $H_{q}(A, B)$.

DeFinition 2.6. Let $u$ be the critical point of functional $J$ defined on Hilbert space E, we denote Morse index of $J$ at $u$ by $\operatorname{ind}(J, u)$,

$$
\operatorname{ind}(J, u) \triangleq \operatorname{dim} \mathrm{E}^{-}, \mathbb{E}^{-}=\left\{\varphi \in \mathrm{E} \mid\left\langle J^{\prime \prime}(u) \varphi, \varphi\right\rangle<0\right\}
$$

\section{Proofs of Theorem 1.1 and Proposition 1.2}

Proof of TheOrem 1.1. By using the excision property, we obtain $C_{q}\left(J, u_{3}\right) \cong H_{q}\left(J^{c_{3}} \cap B\left(u_{3}, r\right), J^{c_{3}} \cap B\left(u_{3}, r\right) \backslash\left\{u_{3}\right\}\right) \cong H_{q}\left(J^{c_{3}}, J^{c_{3}} \backslash\left\{u_{3}\right\}\right) \cong \delta_{q 1} G$. 
Since $H_{q}\left(J^{c_{3}} \backslash\left\{u_{3}\right\}\right) \cong H_{q}(A) \cong \delta_{q 0}(G \oplus G)$, where $A=\left\{u_{1}\right\} \cup\left\{u_{2}\right\}$, by the following exactness of singular homology groups

$$
\begin{aligned}
\cdots \rightarrow H_{q}\left(J^{c_{3}} \backslash\left\{u_{3}\right\}\right) \stackrel{i_{*}}{\longrightarrow} H_{q}\left(J^{c_{3}}\right) \stackrel{j_{*}}{\longrightarrow} H_{q}\left(J^{c_{3}}, J^{c_{3}} \backslash\left\{u_{3}\right\}\right) \\
\stackrel{\partial}{\longrightarrow} H_{q-1}\left(J^{c_{3}} \backslash\left\{u_{3}\right\}\right) \rightarrow \cdots
\end{aligned}
$$

where $i: J^{c_{3}} \backslash\left\{u_{3}\right\} \rightarrow J^{c_{3}}$ and $j: J^{c_{3}} \rightarrow\left(J^{c_{3}}, J^{c_{3}} \backslash\left\{u_{3}\right\}\right)$ are the inclusions, and $\partial$ is the boundary operator. Then we get

$$
H_{q}\left(J^{c_{3}}\right) \cong H_{q}\left(J^{c_{3}}, J^{c_{3}} \backslash\left\{u_{3}\right\}\right) \cong 0, \quad q \geq 2 .
$$

Notice the exactness of singular homology groups

$$
\begin{aligned}
\cdots \rightarrow & H_{1}\left(J^{c_{3}} \backslash\left\{u_{3}\right\}\right) \stackrel{i_{*}}{\longrightarrow} H_{1}\left(J^{c_{3}}\right) \stackrel{j_{*}}{\longrightarrow} H_{1}\left(J^{c_{3}}, J^{c_{3}} \backslash\left\{u_{3}\right\}\right) \\
& \longrightarrow H_{0}\left(J^{c_{3}} \backslash\left\{u_{3}\right\}\right) \stackrel{i_{*}}{\longrightarrow} H_{0}\left(J^{c_{3}}\right) \stackrel{j_{*}}{\longrightarrow} H_{0}\left(J^{c_{3}}, J^{c_{3}} \backslash\left\{u_{3}\right\}\right) \rightarrow \cdots
\end{aligned}
$$

From (3.1) we derive

$\operatorname{rank} H_{1}\left(J^{c_{3}}\right)-\operatorname{rank} H_{1}\left(J^{c_{3}}, J^{c_{3}} \backslash\left\{u_{3}\right\}\right)+\operatorname{rank} H_{0}\left(J^{c_{3}} \backslash\left\{u_{3}\right\}\right)-\operatorname{rank} H_{0}\left(J^{c_{3}}\right)=0$

so we have

$$
\operatorname{rank} H_{0}\left(J^{c_{3}}\right)=1+\operatorname{rank} H_{1}\left(J^{c_{3}}\right) .
$$

Now we will show that $J^{c_{3}}$ is bounded in E. As a matter of fact, since $J$ is bounded from below on $\mathrm{E}$ and satisfies (PS) condition, we infer that $J$ is coercive on E, that is, $J\left(u_{n}\right) \rightarrow \infty$ as $\left\|u_{n}\right\|_{\mathrm{E}} \rightarrow \infty$.

If $J^{c_{3}}$ is not path-connected, we will verify that $u_{1}$ and $u_{2}$ are not in the same component of $J^{c_{3}}$. Otherwise, we assume that they are in the same component of $J^{c_{3}}$, denoted by $A_{1}$. So we can find a path $\Gamma^{*} \subset A_{1}$ connecting $u_{1}$ with $u_{2}$. We define $c \triangleq \inf _{h \in \Gamma} \max _{t \in[0,1]} J(h(t))$, where $\Gamma=\{h \in C([0,1], \mathrm{E}) \mid h(0)=$ $\left.u_{1}, h(1)=u_{2}\right\}$.

From the definition of $c$ and above discussion we infer that

$$
c=c_{3}=\max _{u \in \Gamma^{*}} J(u) .
$$

If $J^{c_{3}}$ is not path-connected, we can find a component of $J^{c_{3}}$, denoted by $A_{2}$, such that $u_{1}, u_{2} \notin A_{2}$.

Consider the pseudo-gradient flow on $J^{-1}\left[c_{1}, c_{3}\right]$ :

$$
\left\{\begin{array}{l}
\dot{\sigma}(t)=-\eta(\sigma(t)) /\|\eta(\sigma(t))\|^{2} \\
\sigma(0)=u \in A_{2}
\end{array}\right.
$$

We claim that $A_{2} \cap K \neq \emptyset$, where $K=\left\{u \in \mathrm{E} \mid J^{\prime}(u)=0\right\}$. If it is not true, fixing $u_{0} \in \AA_{2}$, then for all $t>0, \sigma\left(t, u_{0}\right) \in A_{2}$. Notice that

$$
J\left(\sigma\left(t, u_{0}\right)\right)-J\left(u_{0}\right)=\int_{0}^{t}\langle d J(\sigma(\tau)), \dot{\sigma}(\tau)\rangle d \tau<-\frac{t}{4}
$$


which contradicts $J\left(u_{1}\right)=\inf _{u \in \mathrm{E}} J(u)>-\infty$. So we get a critical point $u_{4}$ in $A_{2}$. Obviously, by (3.2) we get $u_{4} \neq u_{3}$ this is because that $\Gamma^{*} \subset A_{1} \subset J^{c_{3}}$ such that $u_{3} \in A_{1}$. However, it is impossible since we assume that there are only three critical points $u_{1}, u_{2}, u_{3}$ in $J^{c_{3}}$. So we draw the conclusion that if $J^{c_{3}}$ is not path-connected then $u_{1}$ and $u_{2}$ are not in the same component of $J^{c_{3}}$.

Now we will show that $\operatorname{rank} H_{0}\left(J^{c_{3}}\right) \leq 2$. If the thesis is false, let $u_{1} \in \widetilde{A}_{1}$, $u_{2} \in \widetilde{A}_{2}$, where $\widetilde{A}_{1}, \widetilde{A}_{2}$ are two components of $J^{c_{3}}$, so we can find the third component of $J^{c_{3}}$, denoted by $\widetilde{A}_{3}$, such that $u_{1}, u_{2} \notin \widetilde{A}_{3}$. Similar to above discussion we get a critical point $\widehat{u}$ in $\widetilde{A}_{3}$. Obviously, $u_{3} \in \widetilde{A}_{3}$ and $\left(\widetilde{A}_{3}\right)^{\circ} \neq \emptyset$, where $\left(\widetilde{A}_{3}\right)^{\circ}$ denotes the interior of $\widetilde{A}_{3}$. Now we prove that $\widehat{u} \neq u_{3}$. Indeed, we can get a strong result that there exists a local minimizer of $J$ in $\widetilde{A}_{3}$. Define $\widehat{c} \triangleq$ $\inf _{u \in \widetilde{A}_{3}} J(u)$. We will show that there exists a minimizing sequence $\left\{u_{n}\right\} \subset \widetilde{A}_{3}$, $J\left(u_{n}\right) \rightarrow \widehat{c}, J^{\prime}\left(u_{n}\right) \rightarrow 0, n \rightarrow \infty$. As a matter of fact, from the definition of $\widehat{c}$, by Ekeland variational principle, for $\varepsilon_{n}>0$ and $\varepsilon_{n} \rightarrow 0$ as $n \rightarrow \infty$, there exists $u_{n} \in \widetilde{A}_{3}$ such that

$$
\begin{aligned}
J\left(u_{n}\right) & <\inf _{u \in \widetilde{A}_{3}} J(u)+\varepsilon_{n}, \\
J(u) & \geq J\left(u_{n}\right)-\varepsilon_{n} \rho\left(u_{n}, u\right), \quad \text { for all } u \in \widetilde{A}_{3}
\end{aligned}
$$

which implies $J^{\prime}\left(u_{n}\right) \rightarrow 0, n \rightarrow \infty$. Since $J$ satisfies (PS) condition on E, there exists $\widehat{u} \in \widetilde{A}_{3}$ such that $\widehat{c}=J(\widehat{u}), J^{\prime}(\widehat{u})=0$. So we infer that $\widehat{u}$ is a local minimizer of $J$ in $\widetilde{A}_{3}$, which also contradicts the assumption. The proof is complete.

COROLlary 3.1. Under the assumptions of Theorem 1.1, if (b) holds, there exist at least five critical points of $J$ on $\mathrm{E}$.

Proof. It is easy to check that $c>c_{3}$ provided that (b) holds. So we get two local minimizers and two mountain pass points of $J$. We denote $u_{4}$ by another mountain pass point. Notice that $\mathrm{E}$ is contractible, we have $\beta_{0}=1$, $\beta_{1}=\beta_{2}=\ldots=0$, where $\beta_{q}=\operatorname{rank} H_{q}\left(\mathrm{E}, J^{-a}\right), a>0$ large enough.

$$
\sum_{q=0}^{\infty}(-1)^{q} M_{q}=1
$$

where $M_{q}$ are the Morse type numbers for $K=\left\{u \in \mathrm{E} \mid J^{\prime}(u)=0\right\}$. If we assume that there are only four critical points of $J$ on $\mathrm{E}$, then Morse inequality is applied to the bounded from below function $J$ and (3.4) would lose the balance. Thus, the fifth critical point arises from the contradiction and we complete the conclusion.

Now we are in the position to prove Proposition 1.2. 
Proof of Proposition 1.2. From the discussion of [2], we get $C_{d_{l-1}}(J, 0)$ $\neq 0$ under the assumptions (a)-(c) and also $C_{d_{l}}(J, 0) \neq 0$ by the conditions (a), (b), (d). From the assumption we can find two pairs of sub-sup solution of (1.1), that is, $\left\{\varepsilon \varphi_{1}, M_{1}\right\}$ and $\left\{M_{2},-\varepsilon \varphi_{1}\right\}, \varepsilon>0$ small enough. So we get one local minimizer $u_{1}>0$ in order interval $\left[\varepsilon \varphi_{1}, M_{1}\right]$, and another local minimizer $u_{2}<0$ in order interval $\left[M_{2},-\varepsilon \varphi_{1}\right]$. By mountain pass theorem in order interval, we get a sign-changing mountain pass point $u_{3}$. Now we claim that there exists the fourth nontrivial solution in order interval $\left[M_{2}, M_{1}\right]$. We only treat the case under (a)-(c). Otherwise, there are only three nontrivial solutions in $\left[M_{2}, M_{1}\right]$. Then we will show that $J\left(u_{3}\right)<0$. Notice that for all $u \in \mathrm{E}_{2} \cap B(0, \rho) \backslash\{0\}$, $\mathrm{E}_{2}=\operatorname{span}\left\{\varphi_{1}, \varphi_{2}\right\}, \rho$ small enough and $\rho>\varepsilon$, and by (c), we have

$$
\begin{aligned}
J(u) & =\frac{1}{2} \int_{\Omega}|\nabla u|^{2}-\int_{\Omega} F(x, u) \\
& =\frac{1}{2} \int_{\Omega}|\nabla u|^{2}-\frac{\lambda_{2}}{2} \int_{\Omega} u^{2}+\frac{\lambda_{2}}{2} \int_{\Omega} u^{2}-\int_{\Omega} F(x, u)<0
\end{aligned}
$$

So we can always find a path $\widehat{\Gamma}$ connecting $-\varepsilon \varphi_{1}$ with $\varepsilon \varphi_{1}$ in $\mathrm{E}_{2} \cap B(0, \rho)$ such that $0 \notin \widehat{\Gamma}$. Under the pseudo-gradient flow conrtructed by (3.3), we infer that there exists $t_{1}>0$ and $t_{2}>0, \sigma\left(t_{1}, \varepsilon \varphi_{1}\right)=u_{1}, \sigma\left(t_{2},-\varepsilon \varphi_{1}\right)=u_{2}$. So we find a path $\widetilde{\Gamma}$ connecting $u_{1}$ with $u_{2}$ such that $\sup _{u \in \widetilde{\Gamma}} J(u)<0$. Define $c_{3}=J\left(u_{3}\right)$. By Theorem 1.1, we infer that $J^{c_{3}}$ is path-connected and $c=c_{3}<0$.

Notice that $J^{0}$ is a deformation retraction of $\mathrm{E}$ and by Theorem 1.1 we have

$$
H_{q}\left(J^{0} \backslash\{0\}\right) \cong H_{q}\left(J^{c_{3}}\right) \cong \delta_{q 0} G
$$

so, using the excision property, we get

$$
\begin{aligned}
C_{q}(J, 0) \cong H_{q}\left(J^{0} \cap B(0, r)\right. & \left., J^{0} \cap B(0, r) \backslash\{0\}\right) \\
& \cong H_{q}\left(J^{0}, J^{0} \backslash\{0\}\right) \cong H_{q}\left(E, J^{c_{3}}\right) \cong 0, \text { for all } q
\end{aligned}
$$

which contradicts $C_{d_{l-1}}(J, 0) \neq 0$. By using exactly the same techniques and with very similar proofs, we can deal with the case (a), (b), (d). We complete the proof.

Proceeding along the same lines as in the argument of Proposition 1.2, it suffices for us to treat Proposition 1.3. In [4], the authors study the contractibility of level sets of functionals associated with some elliptic boundary value problems by using analysis tools and also gave some application to elliptic boundary value problems in very weak conditions. The methods used by this paper also can discuss the case given in [4]. 


\section{An example of isolated degenerate critical points}

In this part, we consider elliptic equation with Neumann boundary condition:

$$
\begin{cases}-\Delta u+\alpha u=f(x, u) & \text { for } x \in \Omega, \\ \frac{\partial u}{\partial n}=0 & \text { for } x \in \partial \Omega,\end{cases}
$$

$\Omega \subset \mathbb{R}^{\mathbb{N}}$ be a bounded domain with smooth boundary $\partial \Omega$ and $\alpha>0$ is a constant.

The existence of multiple solutions of nonlinear elliptic equation has been widely studied by many mathematicians. However, we must profess that for the elliptic equation, the understanding of qualitative properties of the nontrivial solutions rather than the existence of multiple nontrivial solutions seems to be limited, at least as far as the author is concerned. In this paper we aim to arriving at complete understanding of qualitative properties of the nontrivial solutions of (4.1). The problem is similar to the elliptic sine-Gordon equation so we believe that it derives from profound physical background. The elliptic sine-Gordon equation originates from the static case of the hyperbolic sine-Gordon equation modelling the Josephson junction in superconductivity. However, the elliptic sine-Gordon boundary value problem as studied in the mathematical literature actually has an opposite sign in front of the sine nonlinearity; it models not the "usual" Josephson junction but rather the Josephson $\pi$-junction, which is of contemporary interest to physicists.

Before giving the example, we need the following lemma:

LEMMA 4.1. Under above elliptic problem, for any critical point $u_{0}$ of functional $J$, which is defined on Hilbert space $\mathrm{E}=H^{1}(\Omega), J \in C^{2}(\mathrm{E}, \mathbb{R})$, if ind $\left(J, u_{0}\right)$ $=0$, then $\operatorname{dim} N\left(u_{0}\right) \leq 1, N\left(u_{0}\right) \triangleq\left\{\varphi \in \mathrm{E} \mid\left\langle J^{\prime \prime}\left(u_{0}\right) \varphi, \varphi\right\rangle=0\right\}$.

Proof. From the assumption, $J^{\prime}(u)=u-\mathrm{K} u, u \in \mathrm{E}, \mathrm{K}=(-\Delta+\alpha)^{-1}$, $\mathrm{K}: \mathrm{E} \rightarrow \mathrm{E}$ is a compact operator. Let $u_{0}$ be a critical point of $J$, ind $\left(J, u_{0}\right)=0$. On the one hand, for all $v \in \mathrm{E}$,

$$
\begin{aligned}
\left\langle J^{\prime \prime}\left(u_{0}\right) v, v\right\rangle & =\int_{\Omega}|\nabla v|^{2}-\int_{\Omega} f^{\prime}\left(u_{0}\right) v^{2} \\
& =\langle(-\Delta+\alpha) v, v\rangle_{L^{2}}-\left\langle f^{\prime}\left(u_{0}\right) v, v\right\rangle_{L^{2}} \\
& =\left\langle v-(-\Delta+\alpha)^{-1} f^{\prime}\left(u_{0}\right) v, v\right\rangle_{H^{1}} \geq 0 .
\end{aligned}
$$

On the other hand, we consider the weighted equation:

$$
\begin{cases}(-\Delta+\alpha) u(x)=\lambda f^{\prime}\left(u_{0}\right) u(x) & \text { for } x \in \Omega, \\ \frac{\partial u}{\partial n}=0 & \text { for } x \in \partial \Omega,\end{cases}
$$

Assume $u_{0}$ is a degenerate critical point, then $\lambda=1$ is a eigenvalue of (4.3). Now we claim that $\lambda=1$ is the principle eigenvalue of (4.3). Otherwise, there 
exist $0<\mu<1$ and $w>0$ satisfying (4.3). So we have

$$
\frac{1}{\mu} w=(-\Delta+\alpha)^{-1} f^{\prime}\left(u_{0}\right) w
$$

Combining (4.2) with (4.3),

$$
0 \leq\left\langle w-(-\Delta+\alpha)^{-1} f^{\prime}\left(u_{0}\right) w, w\right\rangle_{H^{1}}<\left\langle\frac{1}{\mu} w-(-\Delta+\alpha)^{-1} f^{\prime}\left(u_{0}\right) w, w\right\rangle_{H^{1}}=0
$$

A contradiction! The proof is complete.

Given the following conditions:

(a) There is a constant $\widetilde{C}>0$ such that

$$
\left|g_{t}^{\prime}(x, t)\right| \leq \widetilde{C}\left(1+|t|^{p-1}\right), \quad t \in \mathbb{R}
$$

where $g(x, t)=f(x, t)-\alpha t, 1<p<2^{*}-1,2^{*}=2 n /(n-2)$, if $n \geq 3$, and $2^{*}=\infty$, if $n=1,2$.

(b) $f \in C^{1}(\mathbb{R}, \mathbb{R})$. There exist sequences $\left\{a_{i}\right\}$ and $\left\{b_{i}\right\}$, where $a_{i}, b_{i} \in \mathbb{R}$, $i=0,1, \ldots$, satisfying $a_{i}>0, b_{i}<0, a_{0}=b_{0}=0$ and $a_{i} \nearrow \infty, b_{i} \searrow$ $-\infty$ as $i \rightarrow \infty$. Moreover, $f(x, t)>\alpha t$, for $t \in\left(a_{i}, a_{i+1}\right), t \in\left(b_{i+1}, b_{i}\right)$ and $f\left(x, a_{i}\right)=\alpha a_{i}, f\left(x, b_{i}\right)=\alpha b_{i}, i=0,1, \ldots$

(c) $f_{t}^{\prime}\left(x, a_{i}\right)=f_{t}^{\prime}\left(x, b_{i}\right)=\alpha, i=1,2, \ldots$

We state our main result:

THEOREM 4.2. Under the hypothesis (a)-(c), (4.1) has only constant solutions $\left\{a_{i}\right\},\left\{b_{i}\right\}, i=0,1, \ldots$ and $C_{q}\left(J, a_{i}\right) \cong 0, C_{q}\left(J, b_{i}\right) \cong 0$, for all $q$.

Proof. First, we will show that (4.1) has no nonconstant positive and negative solution. If the thesis is false, let $u$ be a nonconstant positive solution. So there exists $x_{0} \in \bar{\Omega}, u\left(x_{0}\right)=\min _{x \in \bar{\Omega}} u(x)$. If $u\left(x_{0}\right) \neq a_{i}$, we have

$$
\alpha u\left(x_{0}\right) \geq-\left.\sum_{i=1}^{N} \frac{\partial^{2} u}{\partial x_{i}^{2}}\right|_{x=x_{0}}+\alpha u\left(x_{0}\right)=f\left(u\left(x_{0}\right)\right),
$$

which contradicts condition (b). If there exists some $i$ such that $u\left(x_{0}\right)=a_{i}$, set $u^{*}=u-a_{i}$, then we obtain

$$
\begin{cases}-\Delta u^{*}+\alpha u^{*}=f(x, u)-\alpha a_{i} \geq \alpha\left(u-a_{i}\right) \geq 0 & \text { for } x \in \Omega \\ \frac{\partial u^{*}}{\partial n}=0 & \text { for } x \in \partial \Omega .\end{cases}
$$

By the strong maximum principle, we infer that $u^{*}$ is a constant. A contradiction! Similar to above discussion, we can verify that (4.1) has also no nonconstant negative solution.

Next we will show that (4.1) has no sign-changing solution. Otherwise, there exists a sign-changing solution $\widetilde{u}, m_{1} \triangleq \min _{x \in \bar{\Omega}} \widetilde{u}(x)<0, m_{2} \triangleq \max _{x \in \bar{\Omega}} \widetilde{u}(x)>$ 
0. By (4.4) we infer that there exists some $b_{i_{0}}=m_{1}, i_{0} \in i=1,2, \ldots$ Take $a_{j_{0}}>m_{2}, j_{0} \in i=1,2, \ldots$

Notice that $\left\{b_{i_{0}}, a_{j_{0}}\right\}$ is a pair of sub-sup solution of (4.1). Then we can find an order interval $\left[b_{i_{0}}, a_{j_{0}}\right]=\left\{u \in C_{0}^{1}(\bar{\Omega}) \mid b_{i_{0}} \leq u(x) \leq a_{j_{0}}\right\}$. Obviously, $\widetilde{u} \in$ $\partial\left[b_{i_{0}}, a_{j_{0}}\right]$. By the strong maximum principle we know that $\mathrm{K}=\mathrm{K}_{\mathrm{E}} \mid \mathrm{X}: \mathrm{X} \rightarrow \mathrm{X}$ is strongly preserving so for $v \in \partial \mathrm{P} \backslash\{0\}$ we have

$$
b_{i_{0}}+v-\nabla J\left(b_{i_{0}}+v\right)=\mathrm{K}\left(b_{i_{0}}+v\right) \gg \mathrm{K}\left(b_{i_{0}}\right)=b_{i_{0}} .
$$

That is

$$
b_{i_{0}}+v-\nabla J\left(b_{i_{0}}+v\right) \in b_{i_{0}}+\stackrel{\circ}{\mathrm{P}} \quad \text { for all } v \in \partial \mathrm{P} \backslash\{0\}
$$

which implies that $-\nabla J$ points at $b_{i_{0}}+v$ inside the cone $b_{i_{0}}+\stackrel{\circ}{\mathrm{P}}$. So we infer that

$$
\left[b_{i_{0}}+\partial \mathrm{P} \backslash\{0\}\right] \cap K_{J}=\emptyset
$$

where $K_{J}=\left\{u \in \mathrm{E} \mid J^{\prime}(u)=0\right\}$. However, $\widetilde{u} \in \partial\left[b_{i_{0}}, a_{j_{0}}\right]$, so $\widetilde{u} \in b_{i_{0}}+\partial \mathrm{P} \backslash$ $\{0\}$. This contradicts (4.5). Thus, we have shown that (4.1) has only constant solutions, that is, the two sequences $\left\{a_{i}\right\}$ and $\left\{b_{i}\right\}$ are all the solutions of (4.1).

Now we claim that $C_{q}\left(J, a_{i}\right) \cong 0$, for all $q$. By the assumption (c) and using Lemma 4.1, we derive that $\operatorname{ind}\left(J, a_{i}\right)=0, \operatorname{dim} N\left(a_{i}\right) \leq 1$. So by the shifting theorem we infer that the critical groups $C_{q}\left(J, a_{i}\right)$ must be one of the following three forms:

(1) $C_{q}\left(J, a_{i}\right) \cong \delta_{q 0} G$, for all $q$, which determines that $a_{i}$ is a local minimizer of $J$

(2) $a_{i}$ is a mountain type critical point of $J$, that is, $C_{q}\left(J, a_{i}\right) \cong \delta_{q 1} G$, for all $q$;

(3) $C_{q}\left(J, a_{i}\right) \cong 0$, for all $q$.

Take a constant $m_{i}$ such that $a_{i-1}<m_{i}<a_{i}$. Let

$$
f_{i}(x, t)= \begin{cases}f\left(x, m_{i}\right) & \text { for } t<m_{i}, \\ f(x, t) & \text { for } m_{i} \leq t \leq a_{i+1}, \\ f\left(x, a_{i+1}\right) & \text { for } t>a_{i+1} .\end{cases}
$$

We consider the following equation:

$$
\begin{cases}-\Delta u=f_{i}(x, u) & \text { for } x \in \Omega, \\ \frac{\partial u}{\partial n}=0 & \text { for } x \in \partial \Omega .\end{cases}
$$

It is easy to check that the solution of (4.6) is also the solution of (4.1). Take $a_{i}<\widehat{m}_{i}<a_{i+1}, M_{i+1}>a_{i+1}$. Obviously, $\left\{m_{i}, M_{i+1}\right\}$ and $\left\{\widehat{m}_{i}, M_{i+1}\right\}$ are two pairs of sub-sup solution of (4.6). So we find two order intervals $\left[m_{i}, M_{i+1}\right]$ and $\left[\widehat{m}_{i}, M_{i+1}\right]$. By degree theory

$$
\operatorname{deg}\left(\nabla J_{i},\left[m_{i}, M_{i+1}\right], 0\right)=\operatorname{deg}\left(\nabla J_{i},\left[\widehat{m}_{i}, M_{i+1}\right], 0\right)=1
$$


where

$$
J_{i}(u)=\frac{1}{2} \int_{\Omega}|\nabla u|^{2}-\int_{\Omega} F_{i}(u), \quad F_{i}(u)=\int_{0}^{u} f_{i}(x, t) d t .
$$

Since $a_{i} \in\left[m_{i}, M_{i+1}\right] \backslash\left[\widehat{m}_{i}, M_{i+1}\right]$, we can find ball $B_{r_{i}}\left(a_{i}\right) \subset\left[m_{i}, M_{i+1}\right] \backslash$ $\left[\widehat{m}_{i}, M_{i+1}\right]$ with radius $r_{i}>0$ and we infer that $\operatorname{deg}\left(\nabla J_{i}, B_{r_{i}}\left(a_{i}\right), 0\right)=0$. Otherwise, by Poincare-Hopf formula (see [1])

$$
\operatorname{deg}\left(\nabla J_{i}, B_{r_{i}}\left(a_{i}\right), 0\right)=\sum_{q=0}^{\infty}(-1)^{q} \operatorname{rank} C_{q}\left(J, a_{i}\right) \neq 0
$$

so there must exist a nonconstant solution in $\left[m_{i}, M_{i+1}\right] \backslash\left[\widehat{m}_{i}, M_{i+1}\right]$, which contradicts the fact (4.1) has only constant solutions. We complete the proof. $\square$

\section{REFERENCES}

[1] K. C. Chang, Infinite Dimensional Morse Theory and Multiple Solution Problems, Birkhäuser, Boston, 1993.

[2] S. J. Li, K. Perera and J. B. Su, On the role played by the Fučík spectrum in the determination of critical groups in elliptic problems where the asymptotic limits may not exist, Nonlinear Anal. 49 (2002), 603-611.

[3] S. J. Li AND Z. Q. WANG, Mountain pass theorem in order intervals and multiple solutions for semilinear elliptic Dirichlet problems, J. Anal. Math. 81 (2000), 373-396.

[4] Z. Li. LiU AND S. J. Li, Contractibility of level sets of functionals associated with some elliptic boundary value problems and applications, NoDEA 10 (2003), 133-170.

[5] K. Perera And M. Schechter, Type (II) regions between curves of the Fučik spectrum and critical groups, Topol. Methods Nonlinear Anal. 12 (1998), no. 2, 227-243.

Manuscript received July 9, 2006

ChONG Li

Institute of Mathematics

AMSS, Academia Sinica

Beijing 100080, P.R. CHINA

E-mail address: lichong@amss.ac.cn 